\title{
Fatores determinantes para o desenvolvimento estético em uma prótese total
}

\author{
Lorena Souza Carvalho', Daniela Porto da Cunha Pereira².
}

\begin{abstract}
Resumo: Este artigo buscou apresentar por meio de uma breve e objetiva revisão de literatura os principais fatores determinantes para a confecção de uma prótese total, levando em consideração aspectos estéticos. Este estudo foi realizado por meio de uma revisão da literatura, na qual utilizou-se de livros, periódicos e por artigos científicos. Dos artigos pesquisados, foram incluídos no estudo somente aqueles que tivessem relação com o objetivo desse trabalho, que relataram sobre a abordagem de influência dos fatores contextuais e clínicos para o desenvolvimento de prótese total. Para se alcançar uma estética desejada em uma prótese total, engloba inúmeros fatores e requer uma harmonia com toda a face. Devolver o sorriso do desdentado tem uma grande relação psicológica, melhora consideravelmente a autoestima e autoconfiança e, portanto, é um quesito fundamental para a reabilitação oral do paciente.
\end{abstract}

Palavras-chave: saúde bucal, estética dental, prótese total.

\section{Determining factors for aesthetic development in a total prosthesis}

\begin{abstract}
This article sought to present, through a brief and objective literature review, the main determining factors for the preparation of a total prosthesis, taking into account aesthetic aspects. This study was carried out through a review of the literature, in which books, periodicals and scientific articles were used. Of the researched articles, only those related to the purpose of this study were included in the study, which reported on the influence approach of the contextual and clinical factors for the development of total prosthesis. To achieve a desired aesthetic in a total prosthesis, it encompasses many factors and requires a harmony with the whole face. Returning the smile of the toothless has a great psychological relationship, greatly improves self-esteem and self-confidence and, therefore, is a fundamental question for the oral rehabilitation of the patient.
\end{abstract}

Keywords: oral health, dental aesthetics, total prosthesis.

\footnotetext{
${ }^{1}$ Graduanda em odontologia da Faculdade Independente do Nordeste - FAINOR, Vitória da Conquista, Bahia, Brasil.

E-mail: lorenacarvalho@hotmail.com;

${ }^{2}$ Especialista em Ortodontia e Saúde Coletiva, professora do curso de odontologia da Faculdade Independente do Nordeste - FAINOR, Vitória da Conquista, Bahia, Brasil. E-mail: danielaporto_vcba@hotmail.com.
}

Autor correspondente: Lorena Souza Carvalho (loreenacarvalho@ hotmail.com). Avenida Luiz Eduardo Magalhães, número 1000, Bairro Candeias, Vitória da Conquista - BA, CEP: 45028 - 440. 


\section{Introdução}

Prótese é a substituição de um tecido perdido ou não formado. Em se tratando de próteses odontológicas, as próteses dentárias substituem dentes perdidos e restauram o osso alveolar reabsorvido, proporcionando uma reabilitação funcional e estética. Como a fabricação de próteses dentárias depende de uma série de fatores, é essencial um bom conhecimento da anatomia e fisiologia da cavidade oral do paciente (ALDROVANDI, 1956).

Segundo o comitê da Associação Americana das Escolas Odontológicas, a "Prótese é a ciência e a arte de prover substitutos oportunos para a porção coronária dos dentes, ou para um ou mais dentes que se perderam e para suas partes associadas, de forma a reconstruir as funções perdidas, imagem estética, conforto e a saúde do paciente” (BERNAL et al., 2005).

A restauração estética do desdentado envolve vários fatores, é necessário possuir uma harmonia da prótese com a face do paciente e obtendo um significativo efeito psicológico, melhorando a sua autoestima e autoconfiança e, portanto, é uma parte fundamental do tratamento reabilitador oral (BROADRIBB, 1989).

Aspectos como sexo e formato do rosto possuem relação no que se diz respeito a confecção estética de uma prótese total. Quanto ao sexo feminino, os ângulos incisais possuem contornos arredondados gerando um efeito esférico dos incisivos centrais superiores e incisivos laterais superiores. Quanto ao sexo masculino, os ângulos retos produzem efeito cuboide nesses mesmos dentes. A forma de escolha dos dentes para próteses dentárias depende também do rosto do paciente: um rosto quadrado requer dentes quadrados, um rosto oval utiliza-se dentes ovais e um rosto afunilado sem dentes afilados (CARLI et al., 2013).

A opinião do paciente deve respeitada e ser dada sua devida importância, visto que, o conceito de belo é subjetivo e pessoal, possuindo relações sociais e culturais. Cada tratamento deve ser funcional e individualizado (CARR et al., 2005). E a caracterização deve ser feita de acordo as necessidades de cada paciente (CASTRO et al., 2000).

Sendo assim, este artigo buscou apresentar por meio de uma breve e objetiva revisão de literatura os principais fatores determinantes para a confecção de uma prótese total, levando em consideração aspectos estéticos. 


\section{Metodologia}

Este estudo foi realizado por meio de uma revisão da literatura, na qual utilizou-se de livros, periódicos e por artigos científicos selecionados através de busca no banco de dados do Google acadêmico, Scielo e da Bireme, a partir das fontes Medline e Lilacs, pesquisados entre setembro e novembro de 2017.

A busca nos bancos de dados foi realizada utilizando às terminologias cadastradas nos Descritores em Ciências da Saúde criados pela Biblioteca Virtual em Saúde desenvolvido a partir do Medical Subject Headings da U.S. National Library of Medicine, que permite o uso da terminologia comum em português, inglês e espanhol. As palavras-chave utilizadas na busca foram: saúde bucal, estética dental e prótese total.

Dos artigos pesquisados, foram incluídos no estudo somente aqueles que tivessem relação com o objetivo desse trabalho, que relataram sobre a abordagem de influência dos fatores contextuais e clínicos para o desenvolvimento de prótese total, a escolha dos fatores relevantes na hora da elaboração e aspectos estéticos para a confecção da prótese total.

\section{Confecção de uma prótese total estética}

Para se alcançar uma estética desejada em uma prótese total, engloba inúmeros fatores e requer uma harmonia com toda a face (CURY et al., 1994). Devolver o sorriso do desdentado tem uma grande relação psicológica, melhora consideravelmente a autoestima e autoconfiança e, portanto, é um quesito fundamental para a reabilitação oral do paciente (CUSSIOLI, 2009).

Um grande desafio para a odontologia moderna tem sido criar próteses totais que restauram a estética trazendo conforto para o paciente durante o período de uso. A estética pode ser analisada de maneira subjetiva de acordo com cada um, a função e o conforto dependeram exclusivamente do planejamento assertivo das próteses (DESPLATS, 1998). Para que possamos considerar esteticamente agradável esse arranjo de dentes artificiais deve ser construído de maneira que estabeleça a harmonia no rosto do paciente levando sempre em consideração as variáveis como a cor da pele, o cabelo do indivíduo, os olhos, os traços pessoais, a expressão facial de cada um, e outros detalhes anatômicos individuais. Apesar disso o conjunto da Montagem dos dentes em próteses totais é um tema controversos na literatura, 
ainda que haja avanços em virtude da existência de variadas teorias sobre a sua confecção (ENGELMEIER, 1996).

Em 1990 HEARTWELL e RAHN relataram em seu trabalho que a avaliação artística deve ser respeitada no arranjo dentário de uma prótese total. Requisitos como a cor, alinhamento, disposição, a cor da gengiva artificial, oclusão, estão entre os recursos usados para se ter um aspecto com toque natural e harmonioso. Para um resultado mais satisfatório se faz necessário questionar o paciente a sua preferência para assim saber qual a melhor opção.

A plástica em prótese total é um assunto que aborda diversos elementos que, em conjunto, acomodam o equilíbrio da boca com o restante do rosto. Para a aquisição da estética promissora, é imprescindível observar uma tomada correta do aspecto alinhado, do plano oclusal protético, da série mediana, da série dos caninos e da linha alta do sorriso; com correlação aos dentes, a aparência, a dimensão e a coloração são os elementos mais essenciais a serem considerados para a correta compilação dos dentes artificiais. Além da maneira, a uso dos dentes é um agente indispensável na construção de uma prótese mais agradável, tornandoa mais natural (FREITAS et al., 1974) (FRUSH et al., 1959).

Um outro fator de total valia e que precisa constantemente ser levado em consideração é a seleção de coloração dos dentes que precisa ser escolhida para o paciente. Para esta seleção, deve-se levar em conta elementos importantíssimos, como a coloração de pele, uma vez que quanto mais escura for a pele do paciente, mais escura terá que ser a coloração do dente escolhido. Em pacientes jovens deve-se usar tons mais claros de dentes, e em pacientes mais idosos, por consequência, tons mais escuros (BROADRIBB, 1989).

Em casos de próteses fixas elas também podem ser unitárias quando o dente do paciente houver passado por um processo destrutivo que inutilize sua função, neste caso obrigatoriamente o profissional cirurgião dentista deverá realizar o tratamento endodôntico do canal antes de proceder com a instalação do pino intrarradicular e cimentação da coroa (GOIATO et al., 2005).

A utilização de aparelho dentário nada mais é do que um tratamento para recuperação da estética facial e função mandibular (GOLDSTEIN, 1980). E mesmo com aparecimento e da disseminação das próteses tecnologicamente mais articuladas, as próteses convencionais, totais ou parciais, ainda representam uma alternativa de tratamento importante para devolver função e estética a aqueles pacientes que não podem se submeter a esse tratamento por apresentarem limitações biológicas como doenças, lesões ou inflamações decorrentes de algum problema 
mais profundo, que restringem os procedimentos cirúrgicos exigidos para a sua instalação, ou também por limitações financeiras (GOMEZ et al., 1998) (JOHN et al., 1955).

O tamanho dos dentes é tido pelo tamanho da sua base, e devem estar dentro do limite das linhas de referência demarcadas pelo cirurgião nos roletes de articulação. Método esse proposto por autores que não concordavam com a técnica de utilização do espaço entre as comissuras labiais para determinar a largura dos seis dentes anteriores (LANDA, 1952).

O conjunto de dentes que significa disposição na prótese é um fator de suma importância além dos fatores habituais como forma, cor e tamanho dos dentes postiços assim como são importantes a primeira consulta com o paciente para identificar a expectativa do mesmo, para a tomada da decisão correta sobre a disposição do plano oclusal e a influência da própria instalação e também da manutenção do aparelho (MESQUITA, 2012).

$\mathrm{O}$ elemento personalidade, classificam os indivíduos em tipo potente (se refere a homens quase sempre), médio (a maior parcela populacional), e delicado (mulheres na maioria das vezes) este fator traz harmonização aos contornos faciais de acordo com cada expressão ângulos arredondados harmonizam se com o sexo femininos e os retos com o sexo masculino (NEVILLE et al., 2004).

Em um estudo realizado por NICACIO (1976), 49 universitários dentados, 20 do gênero masculino e 29 do gênero feminino, com idade aproximada de 21 anos, foi feita a comparação das técnicas contidas na literatura para apontar qual método resulta na seleção de dentes artificiais com volumes mais próximas dos dentes naturais e concluiu-se que o conjunto adequado dos dentes aumenta a estabilidade da prótese, o conforto, a estética e a função.

Nota-se a influência de outros fatores na conciliação estética, entre eles estão: a necessidade do entrevistar o paciente no seu primeiro contato com o cirurgião dentista para compreender as expectativas do indivíduo, a tomada necessária da relação Centrica e excêntrica, deste modo localidade do plano oclusal, assim como a influência da própria entrega e conservação do aparelho protético. Elementos cosméticos e a reflexão artística têm que ser considerados no conjunto dentário de uma prótese total (PHOENIX, 2003).

WALLISZEWSKI (2005) sugeriu algumas teorias para seleção do arranjo dentário como por exemplo a tempo a teoria temperamental e Harmonia que se correlaciona com a cor e a forma dos dentes em harmonia com a idade e gênero do indivíduo. Desta maneira buscouse na conjectura de que a personalidade do indivíduo e as suas características corporais e individuais combinavam com o tamanho, disposição, proporção e cor dos dentes artificiais, 
portanto as características individuais do paciente devem sempre estar determinantes proporcionais ao formato estético dos dentes confeccionados.

A desrespeito fonética, pode levar a situações trabalhosas, como dificuldades na fala e distorções de consoantes (WALLISZEWSKI, 2005). Portanto, há muitas formas de utilizar a fonética nas fases clínicas da reabilitação oral de pacientes (NEVILLE et al., 2004). Também é importante ressaltar que que fatores como o diagnóstico e entrevista com o paciente são determinantes para a construção de uma base adequada para prótese seja parcial ou total tornando-se necessário considerar a opinião do indivíduo na escolha do melhor arranjo, pois a prótese deve refletir a personalidade do mesmo e não a do cirurgião-dentista ou do plástico envolvido na montagem, devendo estes ser treinados para perceber as particularidades de cada paciente para não manter um padrão de arranjo dentário que não seja proporcional a face esteticamente falando (PHOENIX, 2003).

Dentes artificiais conseguem também ser construídos em porcelana ou em resina acrílica, e são capazes de ser funcionais ou anatômicos sob o contorno do rosto. Os dentes artificiais fabricados em porcelana são mesclados por quartzo, caulim e feldspato, e leva como corante o ácido metabólico. Estes materiais são fundidos e, em castas, formam o corpo do dente e a porção incisal (PHOENIX, 2003).

O conceito da morfopsicologia deve ser considerado, isto significa, como o ser humano enxerga o outro. De acordo com esse ponto de vista, os incisivos centrais focam os atributos de personalidade individual como, energia, força, autoritarismo, apatia magnetismo ou retração. Os incisivos laterais aliam o abstrato: elementos artísticos, emocionais ou intelectuais da personalidade. Sendo desta forma, o profissional, assim ao confeccionar uma prótese total pode alterar por completo a aparência do indivíduo de forma positiva ou não (BROADRIBB, 1089).

\section{As moldeiras}

Quando queremos uma cópia de um objeto, há a obrigação de efetivarmos uma ação chamada de moldagem. Assim o resultado dessa moldagem, que é o negativo do objeto moldado damos o nome de molde. Dessa forma a inclusão de um material especifico no interior do molde seja ele o gesso, resina acrílica, metal independente de qual, derivará eminentemente em um modelo, que representa a cópia análoga do mesmo. Porém, para que este ato seja feito, 
precisamos de um recipiente para aparar o material moldador durante a realização da moldagem chamado de moldeira (PORTO et al., 2010).

Deste modo, em prótese, moldeiras são recipientes adequados para levar à boca do paciente uma certa quantidade de material de moldagem, antecipadamente manipulado corretamente, distribuindo-o de igual sobre uma área a ser moldada e mantê-lo na mesma posição até seu endurecimento total (PHOENIX, 2003).

\section{Resultados}

De acordo com os dados levantados na pesquisa e apresentados nesta revisão a realização exame clínico deve ser criterioso e registrado no prontuário do paciente, para assim dar sequência ao protocolo clínico (tabela 1). Por meio da anamnese, é possível conhecer a queixa principal do paciente para que, ao final do tratamento, com o aconselhamento do profissional, garantir que essa queixa seja atendida. A anamnese é importante para a análise do perfil psicológico do paciente, o qual está estritamente relacionado com o sucesso do tratamento de reabilitação.

Tabela 1 - protocolo clínico adotado para confecção de próteses totais

\begin{tabular}{|l|l|}
\hline & O protocolo clínico próteses totais removíveis convencionais \\
\hline Passo 1 & $\begin{array}{l}\text { Exame clínico } \\
\text { Moldagem anatômica }\end{array}$ \\
\hline Passo 2 & Moldagem funcional \\
\hline Passo 3 & $\begin{array}{l}\text { Registro das relações maxilo mandibulares } \\
\text { Confecção da base de prova com rolete de cera para fazer os registros } \\
\text { Montagem em Articulador Semi- ajustável (ASA) } \\
\text { Seleção dos dentes artificiais }\end{array}$ \\
\hline Passo 4 & $\begin{array}{l}\text { Prova dos dentes montados em cera } \\
\text { Seleção da cor da gengiva }\end{array}$ \\
\hline Passo 5 & Instalação das próteses e orientações ao paciente \\
\hline Passo 6 & Controles \\
\hline
\end{tabular}

Fonte: Castro Jr. et al

O estudo realizado por Castro Jr. et al obteve se a preferência dos pacientes de acordo com o tipo de montagem sendo importante informar que não houve diferenças nas montagens 
e estéticas em relação a clássica e senil e também entre toque masculino e com diastema, conforme a tabela 2 .

Tabela 2 - Preferência dos pacientes de acordo com o tipo de montagem

\begin{tabular}{|c|c|c|c|c|}
\hline Quantidade & Tipo & $\begin{array}{l}\text { Porcentagem } \\
\text { (\%) aprovação }\end{array}$ & $\begin{array}{l}\text { Porcentagem } \\
(\%) \\
\text { desaprovação }\end{array}$ & $\begin{array}{l}10 \\
\text { voluntários } \\
59 \text { anos } \\
\end{array}$ \\
\hline 1 & Clássica & $38,57 \%$ & - & \\
\hline 5 & Senil & $32,85 \%$ & - & \\
\hline 3 & Toque feminino & - & $32,86 \%$ & \\
\hline 2 & Toque masculino & - & $31,43 \%$ & \\
\hline 4 & Com diastema & - & $22,86 \%$ & \\
\hline
\end{tabular}

Fonte: Castro Jr. et al

\section{Discussão}

Confeccionar próteses totais que restabeleçam a função, a estética e que permitam conforto durante o uso é um grande desafio para o profissional. A estética é avaliada de maneira subjetiva, de acordo com cada indivíduo, e a função e o conforto dependem do correto planejamento das próteses. Para ser considerado esteticamente agradável, o arranjo dos dentes artificiais deve ser tal que promova harmonia facial ao paciente, observando variáveis como a cor da pele, do cabelo e dos olhos, os traços de expressão facial e demais detalhes anatômicos individuais. Contudo, o arranjo na montagem dos dentes em próteses totais ainda é um assunto controverso na literatura em virtude da existência de diferentes teorias para sua realização (TAMAKI, 1983).

Estudos que avaliam o arranjo da montagem dos dentes em prótese total para obterem a satisfação estética do paciente devem considerar que a forma e a aparência dos dentes precisam estar em harmonia com a face. Pesquisas que mostram apenas os arranjos das montagens dos dentes são limitados, embora os arranjos perfeitamente alinhados são os de maior aceitação (TORREÃO, 1999). 


\section{Conclusão}

Os conjuntos dentários são de fato um fator determinante para que haja efetivamente o cumprimento de todos os pré-requisitos necessários para satisfazer os critérios de caráter pessoal, se alterando conforme a vontade e a expectativa de cada pessoa. Inúmeras técnicas, adotando linhas distintas, como teoria dento gênica, teoria temperamental, forma e montagens dos dentes de acordo com a idade de cada indivíduo, que permitem a definição do conjunto que deverá ser criado. Mas, não há uma padronização que seja única para essas opções. Essa escolha estar sujeito a necessidade e a vontade do paciente bem como o bom senso do profissional no momento da escolha sendo de fundamental importância para a satisfação do indivíduo (paciente) a estética em prótese total.

\section{Referências}

ALDROVANDI, C. Dentaduras completas. 2. ed. Rio de Janeiro: Científica, 1956. v. 2, Cap. 6, p. 190205.

BERNAL, C. D. E, CORRÊA, G. D. O, CONTRERAS, E. F. R, SOUZA JÚNIOR, J. A. D: Estética em prótese total. Revista UNINGÁ, v. 2, n.5, p. 107-123, 2005.

BROADRIBB, K. Natural-looking dentures, Dent. Tech, v, 42, n.3, p.12, Mar. 1989.

CARLI, J. P. De et al. Lesões bucais relacionadas ao uso de próteses dentárias removíveis. SALUSVITA, Bauru, v. 32, n. 1, p. 103- 15, 2013.

CARR, A.B, MCGIVENY, G.P.; BROWN, D.T: Removable partial prosthodontics. Missouri: Elsevier Mosby, 11. Ed, 2005.

CASTRO Jr., O. V. de; HVANOV, Z. V.; FRIGERIO, M. L. M. A avaliação estética da montagem dos seis dentes superiores anteriores em prótese total. Pesq. odont bras., v. 14, n. 2, p. 177-82, abr./jun. 2000 .

CURY, A. D. B., RODRIGUES JÚNIOR, A. L; PANZERI, H. Resinas Acrílicas dentais polimerizadas por energia de micro-ondas, método convencional de banho de água e quimicamente ativada: propriedades físicas. Rev Odontol. Univ São Paulo, v. 8, n. 4, p. 243-9, 1994.

CUSSIOLI, B.L. Caracterização de dentes em prótese total, v. p 12, 2009.

DESPLATS, E., KEOGH, T. Protesis parcial removible. $1^{\text {a }}$ ed. Madrid: Harcourt; 1998.

ENGELMEIER, R. L. Complete-denture esthetics. Clin north am, v. 40, n. 1, p. 71-84, jan. 1996. 
FREITAS, A. G., SILVA, N. F., GEOFFROY FILHO, V. M. Prótese total (fases de sua elaboração). Rev bras odont, v. 31, n. 188, p. 155-159, jul. /ago. 1974.

FRUSH, J. P.; FISHER, R. D. Dentogenic, its practical applications. J Prosthet. Dent, 1959.

GOIATO, M.C., CASTELLEONI, L., SANTOS, D.M., GENNARI FILHO, H., ASSUNÇÃO, W.G. Lesões orais provocadas pelo uso de próteses removíveis. Pesq Bras Odontoped Clin Integr, João Pessoa, v. 5, n. 1, p. 85-90, 2005.

GOLDSTEIN, R. E. Estética em odontologia. Rio de janeiro: guanabara koogan, 1980. P. 2-15.

GOMEZ, T., MORI, M., CORRÊA, G. A. Atlas de caracterização em prótese total e prótese parcial removível. São Paulo: santos, 1998. Cap. 3, p. 21.

HEARTWELl Jr., C. M.; Rahn, A. O: Syllabus em Dentaduras Completas. Trad. De José Cerrati Turano e Fernando Montenegro. 4. Ed. São Paulo: Santos. Cap. 14, p. 343-353, 1994.

JOHN P. FRUSH, D.D.S., AND ROLAND D. FISHER, D.D.S. Read before the Academy of Denture Prosthetics in New York, May 5, 1955. Received for publication Feb. 2, 1955

LANDA, J. S. The free-way space and its significance in the rehabilitation of the masticatory apparatus. J. Prosthet. Dent., St. Louis, v.2, n.6, p.756-779, Nov. 1952.

MESQUITA FILHO, J.D. Prótese total (manual de laboratório), p, 4-5, 2012.

NEVILLE, B.W.; DAMM, D.D.; ALLEN, C.N.; BOUQUOT, J.E. Patologia oral e maxilofacial. $2^{\mathrm{a}}$. Ed., Rio de Janeiro: Guanabara Koogan, 2004.

NICACIO, I. M. Considerações sobre a seleção dos dentes artificiais em prótese total. Rev Ass Paul Cirurg Dent, v. 30, p. 358-62, nov. /dez. 1976.

PHOENIX, R. Clinical removable partial prosthodontics. 3a. Ed., Hanover Park: Quintessence Publishing, 2003.

PORTO F. P. A. e al. Protocolo clínico para confecção de próteses removíveis, n. 29, 2010.

TAMAKI, T. Dentaduras completas. 4 ed. São Paulo: Sarvier, 1983, p.232.

TORREÃO, A.C.R. Levantamento epidemiológico de biópsias da região bucomaxilofacial, encaminhadas ao laboratório de patologia bucal da Faculdade de Odontologia de Pernambuco. Rev Cons Reg Odontol Pernambuco, Recife, v. 2, n. 2, p. 118-125, 1999

WALISZEWSKI M.P. Restoring dentate appearance: A literature review for modern complete denture esthetics. Journal of Esthetic and Restorative Dentistry, v. 93, n. 1, p. 386-94, 2005.

\section{Como citar este artigo (Formato ABNT):}

CARVALHO, Lorena S.; PEREIRA, Daniela Porto da C. Fatores determinantes para o desenvolvimento estético em uma prótese total. Id on Line Rev.Mult. Psic., 2018, vol.12, n.40, p.480-489. ISSN: 1981-1179. 